\title{
Provider and clinical setting characteristics associated with tobacco pharmacotherapy dispensed in the Veterans Health Administration
}

\author{
Amanda C. Blok ${ }^{1,2}$, Rosalinda V. Ignacio ${ }^{3,1}$, Mark C. Geraci ${ }^{4}$, Hyungjin Myra Kim ${ }^{5,1}$, Paul G. Barnett ${ }^{6}$, Sonia A. Duffy ${ }^{7,1,8}$
}

\begin{abstract}
INTRODUCTION While initiation rates of tobacco cessation pharmacotherapy have improved both inside and outside the Department of Veteran Affairs (VA), prescribing rates remain low. The objective of this study was to examine correlation of the characteristics of providers, clinics, and facilities with initiation of tobacco cessation pharmacotherapy.

METHODS This retrospective, observational study used VA outpatient electronic medical record data from federal fiscal year 2011. Logistic regression models estimated the adjusted odds ratio associated with provider characteristics for pharmacotherapy initiation.

RESULTS For the 639507 veterans who used tobacco, there were 30388 providers caring for them. Younger $(\mathrm{p}<0.001)$ and female $(\mathrm{p}<0.001)$ providers were more likely to initiate tobacco cessation pharmacotherapy. Compared to physicians, pharmacists were $74 \%$ more likely to initiate pharmacotherapy, while all groups of nurses were $5-8 \%$ and physicians' assistants were $12 \%$ less likely $(\mathrm{p}<0.001)$. Compared to those seen in primary care clinics, patients assessed in substance use treatment clinics were $16 \%$ more likely to have pharmacotherapy initiated $(\mathrm{p}<0.001)$, while those in psychiatry were $10 \%$ less likely $(\mathrm{p}<0.001)$, and those in outpatient surgery were $39 \%$ less likely to initiate pharmacotherapy $(\mathrm{p}<0.001)$. Compared to almost all other classes of VA facilities, patients seen in primary care community-based outpatient clinics (CBOCs) were 7-28\% more likely to initiate pharmacotherapy $(\mathrm{p}<0.0001)$.

CONCLUSIONS While the VA is at the leading edge of providing tobacco cessation pharmacotherapy, targeting quality improvement efforts towards providers, clinics, and facilities with low prescribing rates will be essential to continue the declining rates of tobacco use among VA patients.
\end{abstract}

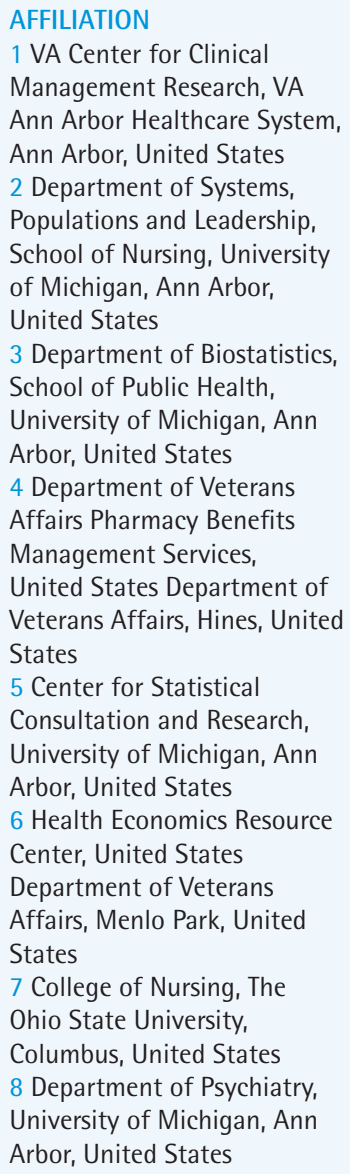

7 College of Nursing, The Ohio State University,

Columbus, United States 8 Department of Psychiatry, University of Michigan, Ann Arbor, United States

CORRESPONDENCE TO Sonia A. Duffy. College of Nursing, The Ohio State University, 1585 Neil Ave, Columbus, OH 43210, United States. E-mail: duffy.278@ osu.edu

KEYWORDS smoking cessation agents, tobacco use cessation, drug prescriptions, health personnel, health services

Received: 30 March 2021 Revised: 4 June 2021 Accepted: 8 July 2021 


\section{INTRODUCTION}

Pharmacological treatment for tobacco dependence has been shown to be clinically effective and cost effective $^{1}$. Thus, clinical practice guidelines have recommended pharmacotherapy for the treatment of tobacco use since $2000^{2}$. In response, the Veterans Administration (VA) implemented the National Smoking and Tobacco Use Cessation Program in 2004, mandating the availability of tobacco cessation pharmacotherapy to veterans who are interested in quitting, regardless of their interest in tobacco treatment programs ${ }^{3}$. This initiative included the implementation of tobacco treatment policy, increased provider training, increased availability of tobacco treatment pharmacotherapy and counseling, and increased the rate and quality of tobacco cessation interventions using clinical reminders in the electronic medical record system.

A prior study by our team showed that tobacco cessation pharmacotherapy for patients in the outpatient setting increased from $13.8 \%$ in 2004 to $26.8 \%$ after a VA Directive was initiated in 2008, then plateaued to $25.8 \%$ in $2011^{4}$. While there was substantial initial improvement, determining practices and providers that remain low-prescribers of pharmacotherapy is needed to break through the plateau effect on prescribing behavior change. This is crucial for tobacco cessation in veterans, as three quarters of those who use tobacco remain untreated. Similarly, studies outside the VA have shown a third or less of tobacco quit attempts are aided by the use of FDA-approved medications ${ }^{5}$. This is unfortunate, as the use of FDA-approved medications increases the quit rate by $50-60 \%$, regardless of setting ${ }^{6}$.

There are many clinical provider-level and clinical setting-level influences that contribute to the low rate of initiating pharmacotherapy for treating tobacco dependence. Clinical providers experience barriers to prescribing pharmacotherapy, which can include a lack of confidence in their ability to provide effective tobacco cessation interventions, false perceptions of a lack of interest by patients, and misconceptions that pharmacotherapy is not effective ${ }^{5}$. In general, providers are far more likely to 'ask' patients about their tobacco status and stop there, rather than continue on to introduce cessation interventions, particularly pharmacotherapy ${ }^{7}$. Clinical settinglevel barriers for prescription initiation can include a lack of time along with competing task pressures for providers, a lack of support for providing tobacco treatment services, a high cost for treatment, or problems with electronic documentation systems ${ }^{8}$.

There is a lack of research describing differences in pharmacotherapy initiation between different types of providers and clinical settings, as well as exploring the influence of these characteristics on pharmacotherapy initiation. Understanding multilevel factors associated with initiating tobacco pharmacotherapy is critical to improve tobacco treatment quality of care, yet requires a large amount of data to do so. This analysis leverages the availability of data from the Veterans Health Administration (VHA), the largest integrated healthcare system in the US, providing care at 170 medical centers and 1074 outpatient sites of care, and serving nine million veterans each year ${ }^{9}$. To target tobacco treatment educational efforts, specific information is needed about the characteristics of providers, clinics and facilities that do and do not initiate tobacco cessation pharmacotherapy. Not only is it important to describe who is currently receiving cessation pharmacotherapy, which our team has explored in the $\mathrm{VA}^{4}$, but also to describe the clinical and organizational attributes that contribute to prescribing pharmacotherapy for tobacco users. Hence, the objective of this study was to determine the characteristics of providers, clinics and facilities that initiated or did not initiate tobacco cessation.

\section{METHODS}

\section{Design}

This was a retrospective, observational study of VA outpatients who used tobacco in federal fiscal year 2011 (FY 2011). Provider, clinic and facility characteristics, as well as patient demographic and clinical data, were abstracted from the VA Corporate Data Warehouse (CDW), the national repository of VA electronic medical records (EMR). Tobacco use status data were obtained from the Health Factor (HF) dataset, containing provider responses to clinical reminders. Full details of the method for determining tobacco use status are available elsewhere ${ }^{4,10}$. Tobacco cessation pharmacotherapy prescription data were abstracted from the outpatient VA Pharmacy Benefits Management (PBM) system. 


\section{Sample}

\section{Patient sample}

The initial cohort of veterans who were patients that used tobacco included 838309 VA outpatients with current tobacco use status in FY 2011 who had at least one hospital or clinic visit in the prior 12 months. Only $13.2 \%$ (765865) of the 5.80 million VA patients in FY 2011 did not have HF data, and therefore tobacco use data, available. To focus on initiation of cessation pharmacotherapy, the study included only those who had not received pharmacotherapy in the prior 12 months ${ }^{11}$. Excluded from the analysis were $23.7 \%$ (198802/838309) of observations that lacked one or more data elements. Reasons for exclusion included inability to identify a single responsible provider at the time of initial screening (14\%), patient in residential setting at time of screening $(2 \%)$, patient record missing data on race $(9.2 \%)$, or patient record missing data on ethnicity (6.3\%). The total of these exceeds $23.7 \%$ because of observations that were missing more than one item. A companion paper indicated that those excluded were significantly less likely to have initiated pharmacotherapy and more likely to be younger, male, non-White, Hispanic, have less psychiatric and medical comorbidities, have died during the follow-up period (all $\mathrm{p}<0.05)^{12}$. The final cohort for analysis included 76.3\% 639507/838309) of Veterans who screened positive for tobacco use and who did not use pharmacotherapy in the prior year.

\section{Provider sample}

Provider initiation (or non-initiation) of tobacco cessation pharmacotherapy was assigned to the provider who conducted the index HF assessment. If multiple providers were involved, the first provider recorded in the $\mathrm{HF}$ assessment was assigned as the responsible provider. While in real-world practice multiple providers may be involved in patients' care, clinician feedback has indicated that the person most likely to initiate tobacco cessation treatment is the person that actually conducts the tobacco cessation assessment. Thus, the person conducting the tobacco assessment was assigned to be the responsible provider. While registered nurses and licensed practical nurses do not have prescription authority, they are able to initiate pharmacotherapy by alerting a prescribing provider and/or adding a prescription to be signed by a prescribing provider. If a responsible provider was not identified in the HF data, responsibility was assigned to the provider of record in the outpatients associated with the index HF assessment.

To align our analyses with the responsible provider, we examined the clinics and facilities where the HF assessment took place to determine the prevalence of tobacco cessation care. Clinic stop codes, standardized codes used to characterize VA clinical settings, were categorized based on the Health Economics and Resource Center's (HERC's) category of care assignments. These codes identify the work group primarily responsible for providing a clinical service and are used for purposes of workload credit, managerial accounting and program evaluation. Details on the linkage is found on the HERC website $^{13}$. Outpatient clinic categories of care with less than $1 \%$ of study patients were combined (dialysis, ancillary services, rehabilitation, diagnostics services, prosthetics, outpatient dental, adult daycare, home care, unassigned). Facility classification, complexity, and rurality, were based on data from the Planning System Support Group (PSSG) ${ }^{14}$.

\section{Procedures}

This study was approved by the Institutional Review Boards (IRBs) at both the VA Ann Arbor Healthcare System and the VA Palo Alto Health Care System. The IRB approved only retrospective use of data from the time of initial IRB approval in 2013. Hence, this study used veterans who were assessed in FY 2011 and followed for 18 months to find the effectiveness of cessation pharmacotherapy ${ }^{12}$.

Pharmacotherapy prescriptions were abstracted from pharmacy data for the 6 months following the index assessment. The provider was deemed to have initiated pharmacotherapy if the patient screened for tobacco use ultimately received a VA prescription for pharmacotherapy within this 6-month period. Predictors of the use of tobacco pharmacotherapy relating to providers, clinics, and facilities, were studied in patients identified as currently using tobacco at the time of their last assessment in 2011. Comorbidities were determined from utilization data in the 12-month period prior to the tobacco assessment in 2011.

\section{Measures}

\section{Outcome variable}

The dependent variable was initiation of tobacco 
cessation pharmacotherapy, including nicotine replacement therapy (NRT), varenicline, and prescriptions for bupropion SR $150 \mathrm{mg}$ taken twice per day, the dose approved by the Food and Drug Administration (FDA) for tobacco cessation. Lower dosages of bupropion, typically used for depression, were not considered cessation pharmacotherapy.

\section{Patient characteristics}

Included as covariates were patient characteristics (age, sex, race, ethnicity, as well as medical, psychiatric, and substance use disorder comorbidities) known to influence patient receptivity and subsequent initiation of tobacco cessation therapy. Standard sets of International Classification of Diseases (ICD9) diagnostic codes were used to identify medical conditions (yes/no $)^{15}$ and psychiatric and substance use disorder comorbidities (yes/no) ${ }^{16}$.

\section{Provider and facility characteristics}

Provider characteristics, included as predictors of patient receipt of cessation pharmacotherapy, included age, sex, provider type (based on position title and degree), clinic (based on the clinic stop), and facility characteristics. Clinics were categorized based on Health Economics Resource Center (HERC) Category of Care Assignments (outpatient primary care, specialty care, psychiatry, substance use disorder treatment, surgery, and other). Facility classification (e.g. VAMC, multi-specialty or primary care community-based outpatient center), complexity, and rurality characteristics were based on VA Site Tracking system (VAST) data from the Planning System Support Group ${ }^{14}$. The Clinical Complexity Index rating for each VAMC complexity relates to the level of patient risk, patient volume and teaching or research done at the facility. The VA Facility Complexity Model classifies VA medical facilities at levels 1a, 1b, 1c, 2, or 3, with level-1a facilities being the most complex and level 3 facilities the least complex. To control for the unmeasured facilitylevel differences in the use of pharmacotherapy and location-specific differences affecting tobacco use, such as tobacco tax rates and tobacco use regulations, a set of indicator variables were created for the 130 facilities where the index tobacco use assessment took place.

\section{Statistical analysis}

The proportion of providers prescribing tobacco cessation pharmacotherapies were calculated overall and by each provider characteristic, clinic classification, and facility characteristic. Logistic regression models were used to estimate the adjusted odds ratio (AOR) for each potential provider characteristic for pharmacotherapy initiation. First, multivariable logistic regression models were done separately for each provider characteristic controlling for patient characteristics (demographic, psychiatric/ substance use diagnosis, medical comorbidities) and facilities, with Generalized Estimating Equation (GEE) controlling for the correlation of patients within providers. Second, multivariable logistic regression model for all provider characteristics controlling for patient characteristics (demographic, psychiatric/ substance use diagnosis, medical comorbidities) and facilities with GEE controlling for the correlation of patients within providers. AORs represent the independent association between a given provider characteristic and pharmacotherapy initiation, i.e. odds of initiation in those with the characteristic relative to those without the characteristic. Provider characteristics of age and sex were missing for 182474 patient-level observations (28.5\%). Due to the problem of using an indicator variable, we performed a multivariable logistic model excluding patients seen by providers with missing age or sex as a sensitivity analysis $(\mathrm{n}=182474 ; 28.5 \%)$.

\section{RESULTS}

The index HF assessment of the 639507 veterans who were patients that screened positive for tobacco use and who did not use pharmacotherapy in the prior year were made by 30388 unique providers in FY 2011 (Table 1). Among providers who made the index $\mathrm{HF}$ assessment, $53 \%$ were age $<55$ years and $52 \%$ were female. Provider types included $47 \%$ physicians, $3 \%$ physician assistants, $10 \%$ nurse practitioners, $17 \%$ registered nurses, 9\% licensed practical/vocational nurses, $1 \%$ pharmacists, and $13 \%$ other providers or missing type data. The majority ( $80 \%$ ) of the patients were assessed in outpatient primary care clinics, $10 \%$ in outpatient psychiatry clinics, $6 \%$ in outpatient specialty clinics, and the remainder in outpatient substance use disorder treatment, outpatient surgery and other outpatient settings (4\%). 
About $49 \%$ of the patients received their tobacco use assessment in VA medical centers (VAMCs) and the majority $(83 \%)$ in VA facilities located in urban areas. Overall, $52 \%$ of providers who made the index HF assessment had one or more patients who initiated tobacco cessation pharmacotherapy.

\section{Table 1. Characteristics of providers caring for veterans who use tobacco}

\begin{tabular}{|c|c|c|c|}
\hline Provider characteristics & $\begin{array}{l}\text { Providers } \\
n(\%)\end{array}$ & $\begin{array}{c}\text { Providers initiating } \\
\text { tobacco cessation } \\
\text { pharmacotherapy a } \\
\%\end{array}$ & $\begin{array}{c}\text { Veterans currently } \\
\text { using tobacco } \\
n(\%)\end{array}$ \\
\hline All & 30388 (100) & 52.5 & 639507 (100) \\
\hline \multicolumn{4}{|l|}{ Age (years) } \\
\hline $18-44$ & 9249 (30.4) & 45.4 & 136037 (21.3) \\
\hline $45-54$ & $6931(22.8)$ & 55.4 & 164835 (25.8) \\
\hline $55-64$ & $6862(22.6)$ & 56.8 & 165609 (25.9) \\
\hline$\geq 65$ & $1403(4.6)$ & 57.6 & 33032 (5.2) \\
\hline$<18$ or missing & $6786(22.3)$ & 51.6 & 139994 (21.9) \\
\hline \multicolumn{4}{|l|}{ Sex } \\
\hline Female & 15907 (52.3) & 51.6 & 307112 (48.0) \\
\hline Male & 9472 (31.2) & 54.1 & 236197 (36.9) \\
\hline Missing & 5009 (16.5) & 51.2 & 96198 (15.0) \\
\hline \multicolumn{4}{|l|}{ Provider type } \\
\hline Physician & $14159(46.6)$ & 57.2 & 382277 (59.8) \\
\hline Physician assistant & $907(3.0)$ & 74.0 & 39399 (6.2) \\
\hline Nurse practitioner & $2959(9.7)$ & 68.2 & $96511(15.1)$ \\
\hline Registered nurse & $5148(16.9)$ & 40.2 & $43874(6.9)$ \\
\hline Licensed practical/vocational nurse & $2802(9.2)$ & 47.6 & $48580(7.6)$ \\
\hline Pharmacist & $398(1.3)$ & 37.7 & $1910(0.3)$ \\
\hline Other & $3755(12.4)$ & 38.4 & 24900 (3.9) \\
\hline Missing & $260(0.9)$ & 39.6 & $2056(0.3)$ \\
\hline \multicolumn{4}{|l|}{ Clinic category } \\
\hline Outpatient medicine-primary care & $16549(54.5)$ & 60.5 & 510765 (79.9) \\
\hline Outpatient medicine-specialty care & $6992(23.0)$ & 31.6 & $37879(5.9)$ \\
\hline Outpatient psychiatry & $6067(20.0)$ & 51.6 & $65946(10.3)$ \\
\hline Outpatient substance use treatment & $964(3.2)$ & 48.1 & $6758(1.1)$ \\
\hline Outpatient surgery & $1182(3.9)$ & 26.2 & $7099(1.1)$ \\
\hline Outpatient other & $2550(8.4)$ & 28.4 & $11060(1.7)$ \\
\hline \multicolumn{4}{|l|}{ Facility classification } \\
\hline Healthcare center & $810(2.7)$ & 54.3 & $16915(2.6)$ \\
\hline Multi-specialty CBOC & 4099 (13.5) & 62.1 & 124009 (19.4) \\
\hline Other outpatient services & $381(1.3)$ & 55.6 & $6456(1.0)$ \\
\hline Primary care $\mathrm{CBOC}$ & $4783(15.7)$ & 62.6 & $143589(22.5)$ \\
\hline VAMC 1a-high complexity & $579(1.9)$ & 43.7 & $15446(2.4)$ \\
\hline VAMC 1b-high complexity & $10188(33.5)$ & 47.0 & 132777 (20.8) \\
\hline VAMC 1c-high complexity & $3813(12.5)$ & 49.6 & $59989(9.4)$ \\
\hline VAMC 2-medium complexity & $2965(9.8)$ & 50.8 & $55585(8.7)$ \\
\hline VAMC 3-low complexity & $2440(8.0)$ & 49.9 & 49640 (7.8) \\
\hline Unclassified & $1765(5.8)$ & 61.1 & $35101(5.5)$ \\
\hline \multicolumn{4}{|l|}{ Facility rurality } \\
\hline Urban & $26822(88.3)$ & 51.1 & 531978 (83.2) \\
\hline Rural/highly rural/insular & 4163 (13.7) & 57.3 & $107529(16.8)$ \\
\hline
\end{tabular}

a For one or more veterans currently using tobacco. СBOC: community-based outpatient clinic. VAMC: Veterans Affairs Medical Center. 
Table 2 presents the results of the logistic regression analysis for pharmacotherapy initiation with provider, clinic, and facility characteristics, as predictors controlling for patient characteristics (demographics, psychiatric conditions, medical comorbidities) and facilities. Results of the multivariable logistic

Table 2. Logistic regression model of treating (or not treating) veterans with tobacco cessation pharmacotherapy ( $N=639507)$; adjusted odds ratios are for odds of pharmacotherapy associated with provider characteristics

\begin{tabular}{|c|c|c|}
\hline Characteristics & $\begin{array}{l}\text { Simple model } \\
\text { AOR }(95 \% \text { CI })^{a}\end{array}$ & $\begin{array}{l}\text { Multivariable model } \\
\text { AOR }(95 \% \text { CI })^{b}\end{array}$ \\
\hline
\end{tabular}

\section{Age (years)}

18-44

$45-54$

$55-64$

$\geq 65$

$<18$ or missing

Sex

Female

Male

Missing

Provider type

Physician

Physician assistant

Nurse practitioner

Registered nurse

Licensed practical/vocational nurse

Pharmacist

Other

Missing

Clinic category

Outpatient medicine-primary care

Outpatient medicine-specialty care

Outpatient psychiatry

Outpatient substance use treatment

Outpatient surgery

Outpatient other

Facility classification

Healthcare center

Multi-specialty CBOC

Other outpatient services

Primary care $\mathrm{CBOC}$

VAMC 1a-high complexity

VAMC 1b-high complexity

VAMC 1c-high complexity

VAMC 2-medium complexity

VAMC 3-low complexity

Unclassified

Facility rurality

Urban

Rural/highly rural/insular
Ref.

$0.96(0.93-0.99)^{* *}$

$0.90(0.87-0.94)^{* *}$

$0.85(0.80-0.90)^{* *}$

$0.99(0.95-1.04)$

Ref.

$0.94(0.92-0.97)^{* *}$

$1.06(1.02-1.10)^{* *}$

Ref.

$0.89(0.84-0.94)^{* *}$

$0.97(0.94-1.01)$

$0.96(0.92-1.00)$

$0.97(0.92-1.02)$

$1.70(1.47-1.97)^{* *}$

$0.85(0.81-0.89)^{* *}$

$1.05(0.88-1.26)$

Ref.

$0.90(0.86-0.94)^{* *}$

$0.86(0.83-0.90)^{* *}$

1.06 (0.97-1.15)

$0.57(0.51-0.64)^{* *}$

$0.91(0.85-0.98)^{*}$

$0.95(0.86-1.04)$

$0.89(0.85-0.93)^{* *}$

0.98 (0.87-1.10)

Ref.

$0.81(0.77-0.85)^{* *}$

$0.77(0.72-0.82)^{* *}$

$0.90(0.84-0.97)^{* *}$

$0.79(0.74-0.85)^{* *}$

$0.71(0.66-0.77)^{* *}$

$0.83(0.76-0.91)^{* *}$

Ref.

$1.05(1.01-1.10)^{* *}$

Ref.

$0.96(0.93-0.99)^{*}$

$0.91(0.88-0.95)^{* *}$

$0.87(0.82-0.93)^{* *}$

$0.96(0.92-1.00)$

Ref.

$0.93(0.91-0.96)^{* *}$

1.01 (0.97-1.06)

Ref.

$0.88(0.83-0.94)^{* *}$

$0.95(0.91-0.99)^{* *}$

$0.95(0.91-0.99)^{*}$

$0.92(0.88-0.97)^{* *}$

$1.74(1.50-2.02)^{* *}$

$0.86(0.81-0.91)^{* *}$

$0.97(0.81-1.16)$

Ref.

$0.94(0.90-0.98)^{* *}$

$0.90(0.87-0.94)^{* *}$

$1.16(1.07-1.27)^{* *}$

$0.61(0.55-0.69)^{* *}$

$0.90(0.83-0.97)^{* *}$

$0.93(0.84-1.03)$

$0.89(0.85-0.92)^{* *}$

$1.00(0.88-1.12)$

Ref.

$0.82(0.78-0.86)^{* *}$

$0.77(0.72-0.82)^{* *}$

$0.91(0.85-0.97)^{* *}$

$0.81(0.75-0.86)^{* *}$

$0.72(0.67-0.78)^{* *}$

$0.83(0.76-0.91)^{* *}$

Ref.

0.97 (0.93-1.01)

a Multivariable logistic regression model done separately for each provider characteristic controlling for patient characteristics (demographic, psychiatric/substance use diagnosis, medical comorbidities) and facilities, with Generalized Estimating Equation (GEE) controlling for the correlation of patients within providers. b Multivariable logistic regression model for all provider characteristics controlling for patient characteristics (demographic, psychiatric/substance use diagnosis, medical comorbidities) and facilities with GEE controlling for the correlation of patients within providers. All analyses controlled for 130 facilities using dummy variables for parent facilities. CBOC: communitybased outpatient clinic. VAMC: Veterans Affairs Medical Center. ${ }^{*} p<0.05,{ }^{* *} p<0.001$. 
regression analysis show that providers who were older $(\mathrm{AOR}=0.96, \mathrm{p}<0.05 ; \mathrm{AOR}=0.91 ; \mathrm{AOR}=0.87$; for age groups $45-54$ years, $55-64$ years, $\geq 65$ years respectively, compared to age group 18-44 years) and male providers $(\mathrm{AOR}=0.93, \mathrm{p}<0.001$ for males, compared to females) were less likely to initiate tobacco pharmacotherapy. Moreover, in terms of provider type, compared to physicians, pharmacists were $74 \%$ more likely to initiate pharmacotherapy $(\mathrm{AOR}=1.74, \mathrm{p}<0.001)$, while selected nurse categories were $5-8 \%$ less likely to initiate treatment $(\mathrm{AOR}=0.92$, $\mathrm{p}<0.001$ to $\mathrm{AOR}=0.95, \mathrm{p}<0.001$ for nurse categories, compared to physicians), and physician assistants were the least likely to initiate pharmacotherapy ( $\mathrm{AOR}=0.88, \mathrm{p}<0.001$ for physician assistants, compared to physicians).

Patients assessed as currently using tobacco in outpatient substance use disorder treatment clinics (about $10 \%$ of sample) were more likely to have cessation pharmacotherapy initiated compared to those seen in outpatient primary care clinics $(\mathrm{AOR}=1.16, \mathrm{p}<0.001)$. Those in other clinic categories were slightly less likely to have pharmacotherapy initiated (Table 2 ) with outpatient surgery over a third less likely to receive pharmacotherapy $(\mathrm{AOR}=0.61$, $\mathrm{p}<0.001)$. Those seen in primary care CBOCs were more likely to initiate pharmacotherapy than other classes of VA facilities such as healthcare centers, multi-specialty CBOCs, and VAMCs, $(\mathrm{p}<0.001)$. The sensitivity analysis excluding 182474 (28.5\%) patients seen by providers with missing age or sex yielded similar results as the main analysis.

\section{DISCUSSION}

After adjusting for patient demographic and clinical characteristics and facilities, this study showed that several provider demographic characteristics were associated with providing tobacco cessation pharmacotherapy. Younger providers were more likely to prescribe tobacco cessation pharmacotherapy, perhaps because tobacco treatment is being increasingly emphasized in educational curricula ${ }^{17}$. Female providers were more likely than males to provide pharmacotherapy. Similarly, the literature has shown that female physicians are more likely to adhere to clinical guidelines ${ }^{18}$, as well as provide general and cancer-specific preventive care, including psychosocial and substance use disorder counseling ${ }^{19}$.
While physician-delivered interventions have been found to increase cessation efforts ${ }^{2,20}$, nurseled and pharmacist-led interventions in various clinical settings have shown an increase in patients being offered pharmacotherapy and an improvement in patient acceptance of pharmacotherapy ${ }^{21,22}$. In this study, pharmacists were more likely than the physician reference group to prescribe tobacco pharmacotherapy. This is most likely due to the pharmacists' role in the VA, as pharmacists are often assigned the responsibility of providing tobacco cessation services. VA pharmacists have generally initiated evidence-based quality improvement efforts, such as obtaining an accurate tobaccouse assessment, initiating tobacco cessation pharmacotherapy, and continued follow-up care. Pharmacists are an ideal provider type to initiate pharmacotherapy support for tobacco cessation, with evidence-based outreach programs having the potential to increase the impact of their work on patient cessation. A trial testing the impact of a pharmacist-led telephone counseling program in primary care significantly increased prescription tobacco cessation medication adherence by $4.1 \%$ compared with usual care, and had a higher number of abstinent smokers at 12 weeks ${ }^{23}$.

Under-prescribing clinical provider groups include physician assistants, advanced practice nurses, registered nurses, and licensed nurses. These groups may have a lack of training on providing tobacco cessation in their educational curricula and on the job $^{24}$. An alternative explanation for the lower rates of treatment by physician assistants may reflect their practice setting (e.g. surgical or specialty care) which may defer tobacco treatment to other specialties such as primary care or pharmacy. VA physician assistants and nurse practitioners in primary care have been shown to provide more cost-efficient care than VA physicians ${ }^{25}$, and potentially have more opportunity for initiation as well, due to their smaller panel size. While physician assistants were the lowest prescribing group and are the smallest of the provider groups, addressing barriers of education ${ }^{26}$ and recognition of the importance of the pharmacotherapy prescription guidelines would likely enhance prescription rates by this group. While advance practice nurses and registered nurses initiated tobacco cessation pharmacotherapy only slightly less than physicians, 
nurse-initiated interventions could potentially be ramped up, as nurses are the largest group of frontline providers and nurse-delivered cessation interventions have been found to be efficacious ${ }^{12}$. Staff training and integration into nurse workflow is needed for nurse initiation at the VA, with programs to assist with follow-up on prescription initiation when patients transition from the inpatient to the outpatient setting ${ }^{27}$.

Tobacco use is highly comorbid with substance use disorder, and our study found patients seen in substance use treatment clinics were more likely to initiate pharmacotherapy compared to those seen in outpatient primary care clinics. Patients with substance use disorder (SUD) are twice as likely to smoke than the general population, with a study reporting $59 \%$ of veterans enrolled in a SUD clinic used tobacco and $86.5 \%$ of these patients were offered treatment for tobacco use on intake ${ }^{28}$. While only $41 \%$ attempted cessation when enrolled in the SUD clinic, evidence shows that while the timing of the quit attempt is crucial, quitting tobacco can increase the odds of continued abstinence from all substances ${ }^{29}$. While tobacco use is also highly comorbid with psychiatric disorders, it was unfortunate to find low prescribing rates in outpatient psychiatric clinics. As might be expected and similar to other studies ${ }^{5}$, prescribing rates were particularly low in outpatient surgery where patients may not be fully conscious, but within the broader context of outpatient surgical care, it might also be a missed opportunity to provide pre-operative teaching regarding tobacco cessation.

Similar to other studies ${ }^{30}$, patients seen in primary care CBOCs were fairly likely to initiate tobacco cessation pharmacotherapy (63\% of their providers), perhaps because cessation counselling has become 'standard of care' in primary care ${ }^{2}$. Higher complexity facilities (1a, 1b, and 1c) were less likely to initiate tobacco cessation pharmacotherapy $(44,47$, and $50 \%$, respectively) perhaps because of multiple competing priorities. An explanation for lower prescriptions in other outpatient clinical settings may be clinical practice norms that defer tobacco cessation pharmacotherapy to primary care. This is a large missed opportunity for effectively addressing tobacco use, as every clinical encounter that assesses tobacco use should be followed with the evidence-based action of pharmacotherapy if not already initiated.
Clinical practice norms and attitudes are modifiable and can be addressed in practice improvement efforts.

\section{Policy implications}

VA data show that tobacco use rates have declined from $33 \%$ of VA patients in 1999 to $14.6 \%$ in $2018^{31}$. Contrary to other real-world studies published outside of the $\mathrm{VA}^{32}$, a recently published study on this cohort conducted by our team showed that pharmacotherapy was effective in the real-world VA setting ${ }^{12}$. A second study found that the delivery of cessation pharmacotherapy was cost effective. Compared to the general public, tobacco use is more prevalent in the military ${ }^{33}$ and, as active duty military members transition to the VA setting, many will be in need of tobacco treatment delivered by providers who are optimally prepared. Targeting tobacco cessation education toward provider subgroups and changing culture in clinics and facilities that are least likely to initiate tobacco cessation pharmacotherapy will be essential to quality improvement efforts in the VA. Additionally, health system changes to leverage technology and workflow to support pharmacotherapy is increasingly needed. These efforts are particularly important due to the VA issuing a directive to ban all tobacco use on all VA medical campuses in late $2019^{34}$.

\section{Strengths and limitations}

The study included a large national sample of over 30000 providers that treated almost 640000 veterans that used tobacco. While the results are only generalizable to the VA setting, there are implications for non-VA settings. The study was able to control for a large number of individual and organizational covariates. However, several potential provider factors were not available, including provider race, tobacco use, and attitudes around prescribing. The EMR may not have captured all tobacco treatment interventions provided (including undocumented pharmacotherapy recommendations) or facility differences that designate a particular type of provider to conduct tobacco cessation such that a lower rate of prescription would be expected by other provider types. Studies are in progress to trigger providers to better document tobacco treatment ${ }^{35}$. While the rationale for including prescriptions up to 6 months after the index visit was chosen to increase the likelihood 
that prescribing rates will not be underestimated (a more 'conservative' approach), it also increases the likelihood that the clinician conducting the index assessment and the clinical setting in which it occurred are not tied directly to the actual prescription of tobacco cessation pharmacotherapy. However, a companion paper reported that, of those who initiated pharmacotherapy, the average time from the clinical reminder assessment to pharmacology treatment was 1.3 months $^{4}$. Additionally, the amount tobacco used per day, type of tobacco use, and the type of pharmacotherapy treatment prescribed, were not present in the data, limiting sub-analyses to inform further tailoring of recommendations. While the VA medical record does not allow for identification of type of tobacco (unless written in notes) at the time, the overwhelming majority of veterans who used tobacco smoked cigarettes ${ }^{36}$.

Although evidence supports that the Health Factors data are reasonably reliable and valid for purposes of identifying tobacco use, similar to other settings, there are inconsistencies among providers in how and when tobacco use is assessed. Even though bupropion doses for depression versus tobacco cessation were distinguished in the same way that has been in other studies ${ }^{37}$, there is a possibility that bupropion prescriptions were misclassified. Given that the nicotine patch, gum and lozenge are available over the counter, and that many veterans receive care both inside and outside of VA, it is possible that some participants may have been receiving tobacco cessation medications from other sources. Lastly, the age of the data (FY 2011) may not represent the latest pharmacotherapy prescribing behavior of clinicians, as provider-level and clinical-setting level characteristics, tobacco treatment policies and medication contraindications may have changed ${ }^{38}$.

\section{CONCLUSIONS}

While the VA is at the leading edge of providing tobacco treatment, as in all systems, there are underperforming subgroups of providers and care settings. This study showed that male, older, and nonpharmacist providers, specialty and outpatient surgery clinics, as well as hospital settings, were less likely to provide tobacco cessation pharmacotherapy. As activeduty military members who use tobacco transition to the VA setting, targeting quality improvement efforts towards providers, clinics and facilities with low cessation pharmacotherapy initiation rates will be essential to continuing the declining rates of tobacco use in the VA.

\section{REFERENCES}

1. U.S. Department of Health and Human Services. Smoking Cessation. A Report of the Surgeon General. U.S. Department of Health and Human Services, Centers for Disease Control and Prevention, National Center for Chronic Disease Prevention and Health Promotion, Office on Smoking and Health; 2020. Accessed March 30, 2021. https://www.hhs.gov/sites/default/files/2020-cessationsgr-full-report.pdf

2. Tobacco Use and Dependence Guideline Panel. Treating Tobacco Use and Dependence: 2008 Update. U.S. Department of Health and Human Services; 2008. Accessed March 30, 2021. https://www.ncbi.nlm.nih. gov/books/NBK63952/\#!po=5.00000

3. Department of Veteran Affairs. National Smoking and Tobacco Use Cessation Program. VHA DIRECTIVE 1056. VHA Publications; 2014. Accessed March 30, 2021. https://www.oklahoma.va.gov/1056_D_2014-02-10.pdf

4. Ignacio RV, Barnett PG, Kim HM, et al. Trends and Patient Characteristics Associated with Tobacco Pharmacotherapy Dispensed in the Veterans Health Administration. Nicotine Tob Res. 2018;20(10):1173-1181. doi:10.1093/ntr/ntx229

5. Srivastava B, Ramsey AT, McIntosh LD, et al. Tobacco Use Prevalence and Smoking Cessation Pharmacotherapy Prescription Patterns Among Hospitalized Patients by Medical Specialty. Nicotine Tob Res. 2019;21(5):631637. doi:10.1093/ntr/nty031

6. Hartmann-Boyce J, Chepkin SC, Ye W, Bullen C, Lancaster T. Nicotine replacement therapy versus control for smoking cessation. Cochrane Database Syst Rev. 2018;5(5):CD000146. doi:10.1002/14651858.CD000146.pub5

7. King BA, Dube SR, Babb SD, McAfee TA. Patientreported recall of smoking cessation interventions from a health professional. Prev Med. 2013;57(5):715-717. doi:10.1016/j.ypmed.2013.07.010

8. Duffy SA, Reeves P, Hermann C, Karvonen C, Smith P. Inhospital smoking cessation programs: what do VA patients and staff want and need? Appl Nurs Res. 2008;21(4):199206. doi:10.1016/j.apnr.2006.11.002

9. US Department of Veterans Affairs. Veterans Health Administration. August 10, 2020. Accessed March 30, 2021. https://www.va.gov/health/

10. Barnett P, Ignacio Rosalinda V, Kim HM, et al. CostEffectiveness of Real World Administration of Tobacco Pharmacotherapy in the Veterans Health Administration. Addiction. 2019;114(8):1436-1445. doi:10.1111/add.14621

11. Cunningham FE, Hur K, Dong D, et al. A comparison of neuropsychiatric adverse events during early 
treatment with varenicline or a nicotine patch. Addiction. 2016;111(7):1283-1892. doi:10.1111/add.13329

12. Duffy SA, Ignacio RV, Kim HM, et al. Effectiveness of tobacco cessation pharmacotherapy in the Veterans Health Administration. Tob Control. 2018;28(5):540-547. doi:10.1136/tobaccocontrol-2018-054473

13. U.S. Department of Veteran Affairs. HERG: Average Cost. 2019. Accessed March 30,2021. https://www.herc. research.va.gov/include/page.asp?id=average-cost

14. U.S. Department of Veteran Affairs. HERC: Other data. 2019. Accessed March 30, 2021. https://www.herc. research.va.gov/include/page.asp?id=other

15. Elixhauser A, Steiner C, Harris DR, Coffey RM. Comorbidity measures for use with administrative data. Medical care. 1998:8-27. doi:10.1097/00005650-199801000-00004

16. Quan H, Sundararajan V, Halfon P, et al. Coding algorithms for defining comorbidities in ICD-9-CM and ICD-10 administrative data. Medical Care. 2005;43(11):11301139. doi:10.1097/01.mlr.0000182534.19832.83

17. Herold R, Schiekirka S, Brown J, et al. Structured smoking cessation training for medical students: a prospective study. Nicotine Tob Res. 2016;18(12):22092215. doi:10.1093/ntr/ntw191

18. Baumhäkel M, Müller U, Böhm M. Influence of gender of physicians and patients on guideline-recommended treatment of chronic heart failure in a cross-sectional study. Eur J Heart Fail. 2009;11(3):299-303. doi:10.1093/eurjhf/hfn041

19. Frank E, Dresner Y, Shani M, Vinker S. The association between physicians' and patients' preventive health practices. CMAJ. 2013;185(8):649-653. doi:10.1503/cmaj.121028

20. Stead LF, Bergson G, Lancaster T. Physician advice for smoking cessation. Cochrane Database Syst Rev. 2008;(2):CD000165. doi:10.1002/14651858.CD000165.pub3

21. Rice VH, Heath L, Livingstone-Banks J, HartmannBoyce J. Nursing interventions for smoking cessation. Cochrane Database Syst Rev. 2017;12(12):CD001188. doi:10.1002/14651858.CD001188.pub5

22. Sui CF, Ming LC. Pharmacist-led Smoking Cessation Services: Current and Future Perspectives, in Neuroscience of Nicotine. Elsevier. 2019:441-449.

23. Gong J, Baker LC, Zou KH, et al. A pragmatic randomized trial comparing telephone-based enhanced pharmacy care and usual care to support smoking cessation. J Manag Care Spec Pharm. 2016;22(12):1417-1425. doi:10.18553/jmcp.2016.22.12.1417

24. Binnal A, Gururaghavendran R, Denny C, Ahmed J, Tallada AK. Tobacco Cessation Scenarios Among Healthcare Profession Students: A Multidisciplinary Study. Asian Pac J Cancer Prev. 2018;19(4):1081-1088. doi:10.22034/APJCP.2018.19.4.1081

25. Morgan PA, Smith VA, Berkowitz TSZ, et al. Impact of physicians, nurse practitioners, and physician assistants on utilization and costs for complex patients. Health Affairs. 2019;38(6):1028-1036. doi:10.1377/hlthaff.2019.00014
26. Kelly CW, Davis JM, DiCocco M. Assessing the current status of tobacco dependence education curricula in US physician assistant programs. Journal of Physician Assistant Education. 2011;22(3):4-14. doi:10.1097/01367895-201122030-00001

27. Katz DA, Stewart K, Paez M, et al. 'Let Me Get You a Nicotine Patch': Nurses' Perceptions of Implementing Smoking Cessation Guidelines for Hospitalized Veterans. Mil Med. 2016;181(4):373-382. doi:10.7205/MILMED-D-15-00101

28. Gass JC, Morris DH, Winters J, VanderVeen JW, Chermack S. Characteristics and clinical treatment of tobacco smokers enrolled in a VA substance use disorders clinic. J Subst Abuse Treat. 2018;84:1-8. doi:10.1016/j.jsat.2017.10.006

29. Substance Abuse and Mental Health Services Administration. Implementing Tobacco Cessation Programs in Substance Use Disorder Treatment Settings: A Quick Guide for Program Directors and Clinicians. HHS Publication No. SMA18-5069QG. U.S. Department of Health and Human Services; 2018. Accessed March 30, 2021. https://store.samhsa.gov/sites/default/files/ d7/priv/sma18-5069qg.pdf

30. Ferketich AK, Khan Y, Wewers ME. Are physicians asking about tobacco use and assisting with cessation? Results from the 2001-2004 national ambulatory medical care survey (NAMCS). Prev Med. 2006;43(6):472-476. doi:10.1016/j.ypmed.2006.07.009

31. Wang ZJ, Dhanireddy P, Prince C, Larsen M, Schimpf M, Pearman G. 2019 Survey of Veteran Enrollees' Health and Use of Health Care. Contract No: VA24517-C-0178. U.S. Department of Veterans Affairs; 2020. Accessed March 30, 2021. https://www.va.gov/ HEALTHPOLICYPLANNING / SOE2019/2019_ Enrollee_Data_Findings_Report-March_2020_508_ Compliant.pdf

32. Alpert HR, Connolly GN, Biener L. A prospective cohort study challenging the effectiveness of population-based medical intervention for smoking cessation. Tob Control. 2013;22(1):32-37. doi:10.1136/tobaccocontrol-2011-050129

33. Institute of Medicine (US) Committee on Smoking Cessation in Military and Veteran Populations. Bondurant S, Wedge R, eds. National Academies Press; 2009. Accessed March 30, 2021. https://www.ncbi.nlm.nih. gov/books/NBK215333/toc/?report=reader

34. Veterans Health Administration. Smoke-Free VA Healthcare Facilities. U. S. Department of Veterans Affairs; 2019. Accessed March 30, 2021. https://www. va.gov/health/smokefree/\#top

35. Bae J, Ford EW, Kharrazi HHK, Huerta TR. Electronic medical record reminders and smoking cessation activities in primary care. Addict Behav. 2018;77:203-209. doi:10.1016/j.addbeh.2017.10.009

36. Odani S, Agaku IT, Graffunder CM, Tynan MA, Armour BS. Tobacco product use among military veterans-United States, 2010-2015. Morbidity and Mortality Weekly Report. 2018;67(1):7-12. doi:10.15585/mmwr.mm6701a2 
37. Smith MW, Chen S, Siroka AM, Hamlett-Berry K. Using policy to increase prescribing of smoking cessation medications in the VA healthcare system. Tob Control. 2010;19(6):507-511. doi:10.1136/tc.2009.035147

38. Shah D, Shah A, Tan X, Sambamoorthi U. Trends in utilization of smoking cessation agents before and after the passage of FDA boxed warning in the United States. Drug Alcohol Depend. 2017;177:187-193. doi:10.1016/j.drugalcdep.2017.03.021
ACKNOWLEDGEMENTS

The project was supported by Merit Review Award (Number 101 HX001764) from the United States Department of Veterans Affairs Health Services Research \&t Development Service of the VA Office of Research and Development. The views expressed in this article are those of the authors and do not necessarily represent the views of the Department of Veterans Affairs. We gratefully acknowledge the contribution of Adam Chow of the VA Health Economics Resource Center, Menlo Park, CA for his help in identifying tobacco use status.

\section{CONFLICTS OF INTEREST}

The authors have each completed and submitted an ICMJE form for disclosure of potential conflicts of interest. The authors declare that they have no competing interests, financial or otherwise, related to the current work. P.G. Barnett reports that his institution received payment from Merit Review Award 101 HX001764 from the U.S. Department of Veterans Affairs Health Services Research \& Development Service.

\section{FUNDING}

This work was supported by the Health Services Research and Development Service of the U.S. Department of Veterans Affairs (Proposal number IIR 14-301) awarded to SD. This funding was in the form of an unrestricted allocation. The sponsor did not directly shape the design or conduct of the study, the collection, management, analysis, and interpretation of the data, or the preparation, review, or approval of the manuscript.

ETHICAL APPROVAL AND INFORMED CONSENT

Ethical approval and informed consent were not required as all data used were from existing databases.

\section{DATA AVAILABILITY}

The data supporting this research cannot be made available for privacy reasons.

\section{AUTHORS' CONTRIBUTIONS}

$A C B, S A D, M C G, R V I, H M K$ and PGB made substantial contributions to the conception, interpretation of analysis and findings, and critical revision for intellectual content. RVI made substantial contributions to analysis and interpretation of data. All authors read and approved the final manuscript.

PROVENANCE AND PEER REVIEW

Not commissioned; externally peer reviewed. 